\title{
State-Insensitive Cooling and Trapping of Single Atoms in an Optical Cavity
}

\author{
J. McKeever, J. R. Buck, A. D. Boozer, A. Kuzmich, H.-C. Nägerl, D. M. Stamper-Kurn, and H. J. Kimble \\ Norman Bridge Laboratory of Physics 12-33, California Institute of Technology, Pasadena, California 91125
}

(Received 4 November 2002; published 3 April 2003)

\begin{abstract}
Single cesium atoms are cooled and trapped inside a small optical cavity by way of a novel far-offresonance dipole-force trap, with observed lifetimes of 2-3 s. Trapped atoms are observed continuously via transmission of a strongly coupled probe beam, with individual events lasting $\simeq 1 \mathrm{~s}$. The loss of successive atoms from the trap $N \geq 3 \rightarrow 2 \rightarrow 1 \rightarrow 0$ is thereby monitored in real time. Trapping, cooling, and interactions with strong coupling are enabled by the trap potential, for which the center-ofmass motion is only weakly dependent on the atom's internal state.
\end{abstract}

DOI: $10.1103 /$ PhysRevLett.90.133602

PACS numbers: 42.50.Vk, 03.67.-a, 32.80.Pj

A long-standing ambition in the field of cavity quantum electrodynamics (QED) has been to trap single atoms inside high- $Q$ cavities in a regime of strong coupling [1]. Diverse avenues have been pursued for creating the trapping potential for atom confinement, including additional far off-resonant trapping beams [2], near-resonant light with $\bar{n} \simeq 1$ intracavity photons [3,4], and single trapped ions in high-finesse optical cavities $[5,6]$, although strong coupling has yet to be achieved for trapped ions. A critical aspect of this research is the development of techniques for atom localization that are compatible with strong coupling, as required for quantum computation and communication [7-12].

In this Letter we present experiments to enable quantum information processing in cavity QED by (1) achieving extended trapping times for single atoms in a cavity while still maintaining strong coupling, (2) realizing a trapping potential for the center-of-mass motion that is largely independent of the internal atomic state, and (3) demonstrating a scheme that allows continuous observation of trapped atoms by way of the atom-field coupling. More specifically, we have recorded trapping times up to $3 \mathrm{~s}$ for single Cs atoms stored in an intracavity far-off resonance trap (FORT) [13], which represents an improvement by a factor of $10^{2}$ beyond the first realization of trapping in cavity QED [2], and by roughly $10^{4}$ beyond prior results for atomic trapping [3] and localization [4] with $\bar{n} \simeq 1$ photon. We have also continuously monitored trapped atoms by way of strong coupling to a probe beam, including observations of trap loss atom by atom over intervals $\simeq 1 \mathrm{~s}$. These measurements incorporate auxiliary cooling beams, and provide the first realization of cooling for trapped atoms strongly coupled to a cavity. Our protocols are facilitated by the choice of a "magic" wavelength for the FORT [14-16], for which the relevant atomic levels are shifted almost equally, thereby providing significant advantages for coherent state manipulation of the atom-cavity system.

A major obstacle to the integration of a conventional red-detuned FORT within the setting of cavity QED is that excited electronic states generally experience a pos- itive ac-Stark shift of comparable magnitude to the negative (trapping) shift of the ground state [13]. This effectively introduces a spatially dependent detuning between the atom and cavity resonances, an unfortunate additional complication [16]. However, due to the specific multilevel structure of cesium, the wavelength $\lambda_{F}$ of the trapping laser can be tuned to a region where both of these problems are eliminated for the $6 S_{1 / 2} \rightarrow 6 P_{3 / 2}$ transition, as illustrated in Fig. 1 [14-16]. Around the magic wavelength $\lambda_{F}=935 \mathrm{~nm}$, the sum of ac-Stark shifts coming from different allowed optical transitions results in the ground $6 S_{1 / 2}$ and excited $6 P_{3 / 2}$ states both being shifted downwards by comparable amounts, $\delta_{6 S_{1 / 2}} \simeq \delta_{6 P_{3 / 2}}$, albeit with small dependence on $\left(F^{\prime}, m_{F^{\prime}}\right)$ for the shifts $\delta_{6 P_{3 / 2}}$.

The task then is to achieve state-independent trapping while still maintaining strong coupling for the $6 S_{1 / 2} \rightarrow$ $6 P_{3 / 2}$ transition. Our experimental setup to achieve this end is schematically depicted in Fig. 2 [2]. Significantly, the cavity has a $\mathrm{TEM}_{00}$ longitudinal mode located nine

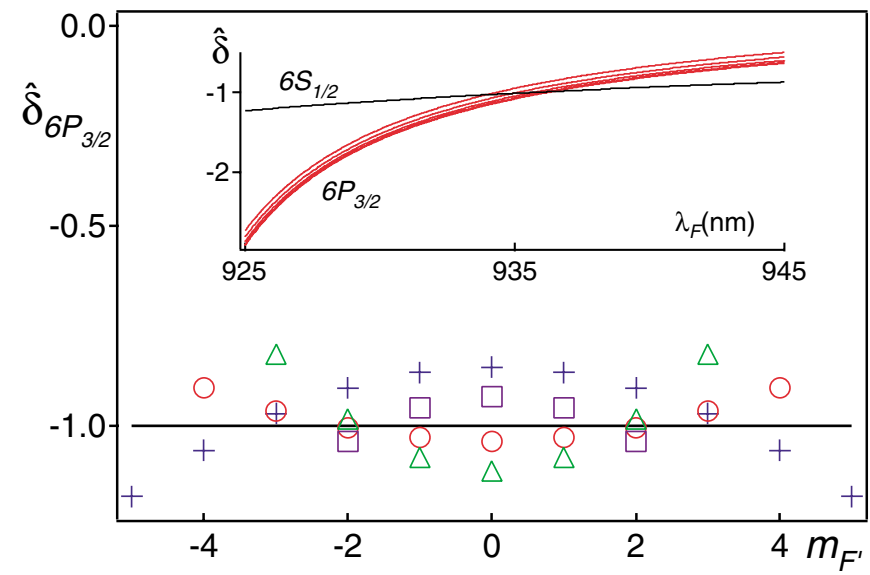

FIG. 1 (color online). ac-Stark shifts $\left(\hat{\delta}_{6 S_{1 / 2}}, \hat{\delta}_{6 P_{3 / 2}}\right)$ for the $\left(6 S_{1 / 2}, 6 P_{3 / 2}\right)$ levels in Cs for a linearly polarized FORT. The inset shows $\left(\hat{\boldsymbol{\delta}}_{6 S_{1 / 2}}, \hat{\boldsymbol{\delta}}_{6 P_{3 / 2}, F^{\prime}=4}\right)$ as functions of wavelength $\lambda_{F}$. The full plot gives $\hat{\delta}_{6 P_{3 / 2}}$ versus $m_{F^{\prime}}$ for each of the levels $6 P_{3 / 2}$, $F^{\prime}=2,3,4,5$ for $\lambda_{F}=935.6 \mathrm{~nm}$. In each case, the normalization is $\hat{\delta}=\delta /\left[\delta_{6 S_{1 / 2}}\left(\lambda_{F}=935.6 \mathrm{~nm}\right)\right]$. 


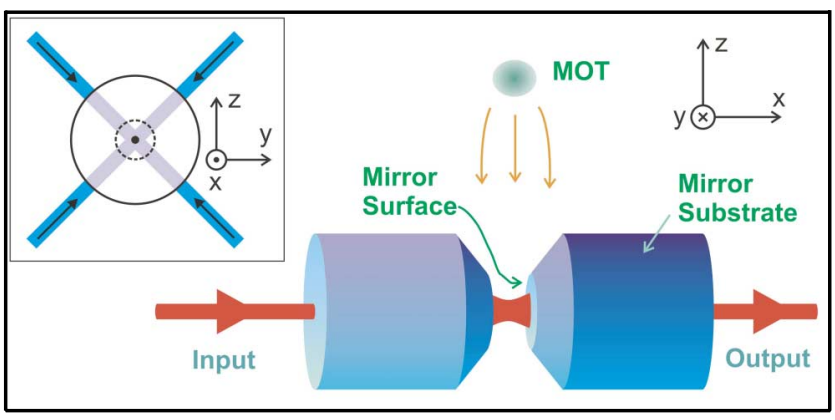

FIG. 2 (color online). Schematic of experiment for trapping single atoms in an optical cavity in a regime of strong coupling. Relevant cavity parameters are length $l=43.0 \mu \mathrm{m}$, waist $w_{0}=23.9 \mu \mathrm{m}$, and finesse $\mathcal{F}=4.2 \times 10^{5}$ at $852 \mathrm{~nm}$. The inset illustrates transverse beams used for cooling and repumping.

mode orders below the mode employed for cavity QED at $852 \mathrm{~nm}$, at the wavelength $\bar{\lambda}_{F}=935.6 \mathrm{~nm}$, allowing the implementation of a FORT with $\delta_{6 S_{1 / 2}} \simeq \delta_{6 P_{3 / 2}}$. The field to excite this cavity mode is provided by a laser at $\bar{\lambda}_{F}$, which is independently locked to the cavity. The finesse of the cavity at $\bar{\lambda}_{F}$ is $\mathcal{F} \sim 2200$ [17], so that a modematched input power of $1.2 \mathrm{~mW}$ gives a peak ac-Stark shift $\delta_{6 S_{1 / 2}} / 2 \pi=-47 \mathrm{MHz}$ for all states in the $6 S_{1 / 2}$ ground manifold, corresponding to a trap depth $U_{0} / k_{B}=$ $2.3 \mathrm{mK}$, which was used for all experiments.

Principal parameters relevant to cavity QED with the system in Fig. 2 are the Rabi frequency $2 g_{0}$ for a single quantum of excitation and the amplitude decay rates $(\kappa, \gamma)$ due to cavity losses and atomic spontaneous emission. For our system, $g_{0} / 2 \pi=24 \mathrm{MHz}, \kappa / 2 \pi=$ $4.2 \mathrm{MHz}$, and $\gamma / 2 \pi=2.6 \mathrm{MHz}$, where $g_{0}$ is for the $\left(6 S_{1 / 2}, F=4, m_{F}=4\right) \rightarrow\left(6 P_{3 / 2}, F^{\prime}=5, m_{F}^{\prime}=4\right)$ transition in atomic Cs at $\lambda_{0}=852.4 \mathrm{~nm}$. Strong coupling is thereby achieved $\left[g_{0} \gg(\kappa, \gamma)\right]$, resulting in critical photon and atom numbers $n_{0} \equiv \gamma^{2} /\left(2 g_{0}^{2}\right) \simeq 0.006, N_{0} \equiv$ $2 \kappa \gamma / g_{0}^{2} \simeq 0.04$. The small transition shifts for our FORT mean that $g_{0}$ is considerably larger than the spatially dependent shift $\delta_{0}$ of the bare atomic frequency employed for cavity QED, $g_{0} \gg \delta_{0} \equiv\left|\delta_{6 P_{3 / 2}}-\delta_{6 S_{1 / 2}}\right|$, whereas in a conventional FORT, $\delta_{0} \sim 2\left|\delta_{6 S_{1 / 2}}\right| \gg g_{0}$.

In addition to the FORT field, the input to the cavity consists of probe and locking beams, all of which are directed to separate detectors at the output. The transmitted probe beam is monitored using heterodyne detection, allowing real-time detection of individual cold atoms within the cavity mode [18]. The cavity length is actively controlled using a cavity resonance at $\lambda_{C}=$ $835.8 \mathrm{~nm}$, so the length is stabilized and tunable independently of all other intracavity fields [2]. The probe as well as the FORT beam are linearly polarized along a direction $\hat{l}_{+}$orthogonal to the $x$ axis of the cavity [17,19].

Cold atoms are collected in a magneto-optical trap (MOT) roughly $5 \mathrm{~mm}$ above the cavity mirrors and then released after a stage of sub-Doppler polarization- gradient cooling [13]. Freely falling atoms arrive at the cavity mode over an interval of about $10 \mathrm{~ms}$, with kinetic energy $E_{K} / k_{B} \simeq 0.8 \mathrm{mK}$, velocity $v \simeq 0.30 \mathrm{~m} / \mathrm{s}$, and transit time $\Delta t=2 w_{0} / v \simeq 150 \mu \mathrm{s}$. Two additional orthogonal pairs of counterpropagating beams in a $\sigma^{+}-$ $\sigma^{-}$configuration illuminate the region between the cavity mirrors along directions at $\pm 45^{\circ}$ relative to $\hat{y}, \hat{z}$ (the " $y-z$ beams") and contain cooling light tuned red of $F=4 \rightarrow F^{\prime}=5$ and repumping light near the $F=3 \rightarrow$ $F^{\prime}=3$ transition [20]. These beams eliminate the freefall velocity to capture atoms in the FORT and provide for subsequent cooling of trapped atoms.

We employed two distinct protocols to study the lifetime for single trapped atoms in our FORT: (1) Trapping in the dark with the atom illuminated only by the FORT laser at $\bar{\lambda}_{F}$ and the cavity-locking laser at $\lambda_{C}$. For this protocol, strong coupling enables real-time monitoring of single atoms within the cavity for initial triggering of cooling light and for final detection. (2) Trapping with continuous observation of single atoms with cavity probe and cooling light during the trapping interval. In this case, atoms in the cavity mode are monitored by way of the cavity probe beam, with cooling provided by the auxiliary $y-z$ beams.

(1) In our first protocol, the $F=4 \rightarrow F^{\prime}=5$ transition is strongly coupled to the cavity field, with zero detuning of the cavity from the bare atomic resonance, $\Delta_{C} \equiv$ $\omega_{C}-\omega_{4 \rightarrow 5}=0$. In contrast to Ref. [2], here the FORT is $O N$ continuously without switching, which makes a cooling mechanism necessary to load atoms into the trap. The initial detection of a single-atom falling into the cavity mode is performed with the probe beam tuned to the lower sideband of the vacuum-Rabi spectrum $\left(\Delta_{p}=\right.$ $\omega_{p}-\omega_{4 \rightarrow 5}=-2 \pi \times 20 \mathrm{MHz}$ ). The resulting increase in transmitted probe power when an atom approaches a region of optimal coupling [21,22] triggers $O N$ a pulse of transverse cooling light from the $y-z$ beams, detuned $41 \mathrm{MHz}$ red of $\omega_{4 \rightarrow 5}$. During the subsequent trapping interval, all near-resonant fields are turned $O F F$ (including the transverse cooling light). After a variable delay $t_{T}$, the probe field is switched back $O N$ to detect whether the atom is still trapped, now with $\Delta_{p}=0$.

Data collected in this manner are shown in Fig. 3(a), which displays the conditional probability $P$ to detect an atom given an initial single-atom triggering event versus the time delay $t_{T}$. The two data sets shown in Fig. 3(a) yield comparable lifetimes, the upper acquired with mean intracavity atom number $\bar{N}=0.30$ atoms and the lower with $\bar{N}=0.019$ [23]. The offset in $P$ between these two curves arises primarily from a reduction in duration $\delta t$ of the cooling pulses, from 100 to $5 \mu \mathrm{s}$, which results in a reduced capture probability. Measurements with constant $\delta t$ but with $\bar{N}$ varied by adjusting the MOT parameters allow us to investigate the probability of trapping an atom other than the "trigger" atom and of capturing more than one atom. For example, with $\delta t=5 \mu$ s as in the lower set, 

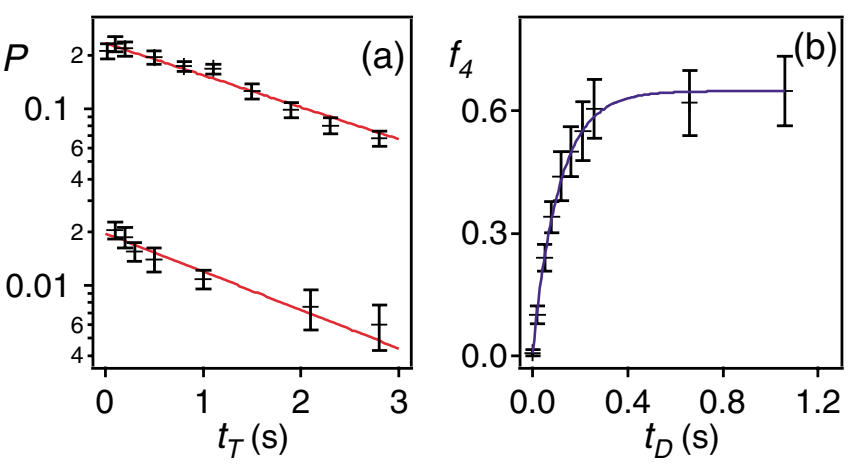

FIG. 3 (color online). (a) Detection probability $P$ as a function of trapping time $t_{T}$. The upper data set is for mean intracavity atom number $\bar{N} \approx 0.30$, while the lower set is for $\bar{N} \approx 0.019$ atoms. Exponential fits (solid lines) yield lifetimes $\tau_{\text {upper }}=(2.4 \pm 0.2) \mathrm{s}$ and $\tau_{\text {lower }}=(2.0 \pm 0.3) \mathrm{s}$. (b) The fractional population $f_{4}\left(t_{D}\right)$ in $F=4$ following depletion of this level at $t_{D}=0$. An exponential fit (solid line) gives $\tau_{R}=$ $(0.11 \pm 0.02) \mathrm{s}$.

we have varied $0.011 \lesssim \bar{N} \lesssim 0.20$ with no observable change in either $P_{T}$ or the trap lifetime $\tau$. Since a conservative upper bound on the relative probability of trapping a second atom is just $\bar{N} / 2$ (when $\bar{N} \ll 1$ ), these data strongly support the conclusion that our measurements are for single trapped atoms. We routinely observe lifetimes $2 \mathrm{~s}<\tau<3 \mathrm{~s}$ depending upon the parameters chosen for trap loading and cooling.

Figure 3(b) explores scattering processes within the FORT that transfer population between the $6 S_{1 / 2}, F=$ $(3,4)$ ground-state hyperfine levels. For these measurements, the $F=4$ level is initially depleted, and then the population in $F=4$ as well as the total $3+4$ population are monitored as functions of time $t_{D}$ to yield the fractional population $f_{4}\left(t_{D}\right)$ in $F=4$. The measured time $\tau_{R}=(0.11 \pm 0.02) \mathrm{s}$ for reequilibration of populations between $F=(3,4)$ agrees with a numerical simulation based upon scattering rates in our FORT, which predicts $\tau_{R}=0.10 \mathrm{~s}$ for atoms trapped at the peak FORT intensity in an initially unpolarized state in the $F=3$ level.

Turning next to the question of the mechanisms that limit our FORT lifetime, we recall that parametric heating can be caused by intensity fluctuations of the trapping field $[2,24]$. From measurements of intensity fluctuations for our FORT around twice the relevant harmonic frequencies $\left(\nu_{\text {axial }}=570, \nu_{\text {radial }}=4.8\right) \mathrm{kHz}$, we estimate a lower bound to the FORT lifetime of $\tau_{p}^{\text {axial }}>1.6 \mathrm{~s}$ [25]. Although this estimate suggests that parametric heating could be a limiting factor in Fig. 3, we were later able to reduce the noise, giving a new lower bound $\tau_{p}^{\text {axial }}>9 \mathrm{~s}$. Unfortunately, the measured FORT lifetime increased only to $\tau=(3.1 \pm 0.4) \mathrm{s}$, indicating that other mechanisms are partially responsible for the observed decay.

A second suspect is a heating process described by Corwin et al. [26] associated with inelastic Raman scat- tering in an elliptically polarized FORT field [19]. We calculate rates $\Gamma_{s}$ for spontaneous Raman scattering in our FORT to be 2.5 to $7 \mathrm{~s}^{-1}$ for transitions that change the hyperfine quantum number $F$, and between 0.8 and $2.5 \mathrm{~s}^{-1}$ when only $m_{F}$ changes [27]. Based on Eq. 3 in Ref. [26] (a two-state model), we estimate an upper limit to the heating rate from this mechanism, $\Gamma_{I R} \lesssim 0.2 \Gamma_{s}$, giving heating times as short as $0.7 \mathrm{~s}$ for the fastest calculated scattering rate. However, we have also undertaken a full multilevel simulation of the optical pumping processes, which indicates much slower heating, $\Gamma_{I R} \sim$ $0.02 \mathrm{~s}^{-1}$. We are working to resolve this discrepancy.

A third suspect that cannot be discounted is the presence of stray light, which we have endeavored to eliminate. For lifetimes as in Fig. 3, we require intracavity photon number $\bar{n} \ll 10^{-5}$, which is not trivial to diagnose. A final concern is the background pressure in the region of the FORT. Although the chamber pressure is $3 \times 10^{-10}$ Torr (leading to $\tau \simeq 30 \mathrm{~s}$ ), we have no direct measurement of the residual gas density in the narrow cylinder between the mirror substrates (diameter $1 \mathrm{~mm}$ and length $43 \mu \mathrm{m}$ ), except for the trap lifetime itself.

(2) Toward the goals of continuous observation of single trapped atoms $[3,4]$ and of implementing $\Lambda$ schemes in cavity QED [7-9,28], we next present results from our second protocol. Here the $F=4 \rightarrow F^{\prime}=4$ transition is strongly coupled to the cavity field, with $\Delta_{C}^{\prime} \equiv \omega_{C}-$ $\omega_{4 \rightarrow 4}=0$. In contrast to our protocol (1), the FORT and the transverse $y-z$ beams are left $O N$ continuously, with the latter containing only light near the $F=3 \rightarrow F^{\prime}=3$ resonance, with detuning $\Delta_{3}$. Significantly, we observe trap loading with no cooling light near the $F=4 \rightarrow$ $F^{\prime}=5$ transition.

An example of the resulting probe transmission is shown in Fig. 4, which displays two separate records of the continuous observation of trapped atoms. Here the probe detuning $\Delta_{p}^{\prime}=\omega_{p}-\omega_{4 \rightarrow 4}=0$ and the probe strength is given in terms of $\bar{m}=|\langle\hat{a}\rangle|^{2}$ deduced from the heterodyne current, with $\hat{a}$ as the annihilation operator for the intracavity field. We believe that the $y-z$ repumping beams (which excite $F=3 \rightarrow F^{\prime}=3$ ) provide cooling, since without them the atoms would "roll" in and out of the near-conservative FORT potential (indeed no trapping occurs in their absence). In addition, this is a continuous cooling and loading scheme, so that we routinely load multiple atoms into the trap.

The most striking characteristic of the data collected in this manner is that $\bar{m}$ versus $t$ always reaches its deepest level within the $\simeq 10 \mathrm{~ms}$ window when the falling atoms arrive, subsequently increasing in a discontinuous "staircase" of steps. As indicated in Fig. 4, our interpretation is that there is a different level for $\bar{m}$ associated with each value $N$ of the number of trapped atoms (with the level decreasing for higher $N$ ), and that each step is due to the loss of an atom from the cavity mode. In addition, we observe a strong dependence both of the initial trapping 


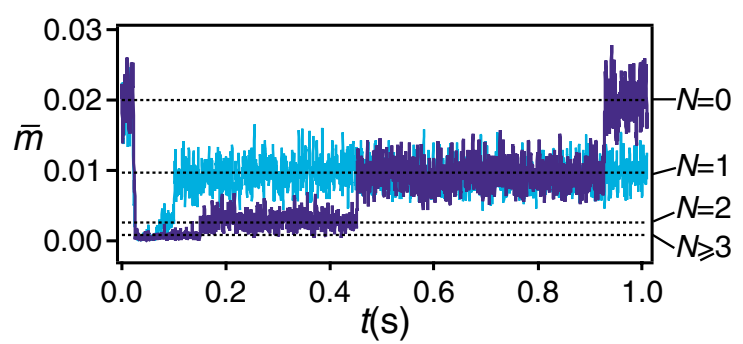

FIG. 4 (color online). Two traces of the continuous observation of trapped atoms inside a cavity in a regime of strong coupling. After an initial sharp reduction around $t=0$ as atoms are cooled into the cavity mode, the intracavity field strength $\bar{m}$ increases in a discontinuous fashion as trapped atoms escape from the cavity mode one by one. rf detection bandwidth $=1 \mathrm{kHz}, \Delta_{C}^{\prime}=0=\Delta_{p}^{\prime}$, and $\Delta_{3} / 2 \pi=25 \mathrm{MHz}$.

probability and of the continuous observation time on the detuning of the transverse beams, with an optimal value $\Delta_{3} \simeq 25 \mathrm{MHz}$ to the blue of the $3 \rightarrow 3$ transition, which strongly suggests blue Sisyphus cooling [29].

We stress that observations as in Fig. 4 are made possible by strong coupling in cavity QED, for which individual intracavity atoms cause the displayed changes in probe transmission. While $\bar{m}$ in Fig. 4 is only $\simeq 0.01$, it represents an output flux $\simeq 5 \times 10^{5}$ photons per second. The probe is also critical to the cooling, although it is not clear whether this beam is acting as a simple "repumper" [29] or is functioning in a more complex fashion due to strong coupling. We have not seen such striking phenomena under similar conditions for cavity QED with the $F=$ $4 \rightarrow F^{\prime}=5$ transition. Note that our ability to monitor the atom as well as to cool its motion are enabled by the state-insensitive character of the trap, since the net transition shifts are small, $\left(g_{0}, \Delta_{3}\right) \gg \delta_{0}$.

In summary, we have demonstrated a new set of ideas within the setting of cavity QED, including stateinsensitive trapping suitable for strong coupling. Trapping of single atoms with $g_{0} \gg\left(\delta_{0}, \kappa, \gamma\right)$ has been achieved with lifetimes $\tau \simeq 2-3 \mathrm{~s}$. Since intrinsic heating in the FORT is quite low $(\sim 11 \mu \mathrm{K} / \mathrm{s}$ due to photon recoil), we anticipate extensions to much longer lifetimes. Continuous observations of multiple atoms in a cavity have been reported, and involve an interplay of a strongly coupled probe field for monitoring and a set of $y-z$ cooling beams. Our measurements represent the first demonstration of cooling for trapped atoms strongly coupled to a cavity. Beyond its critical role here, stateinsensitive trapping should allow the application of diverse laser cooling schemes, leading to further advances in quantum information science.

We gratefully acknowledge the contributions of K. Birnbaum, A. Boca, T.W. Lynn, S. J. van Enk, D.W. Vernooy, and J. Ye. This work was supported by the Caltech MURI Center for Quantum Networks under
ARO Grant No. DAAD19-00-1-0374, by the National Science Foundation, and by the Office of Naval Research.

[1] For a review, see Phys. Scr. T76, 127 (1998).

[2] J. Ye et al., Phys. Rev. Lett. 83, 4987 (1999); D.W. Vernooy, Ph.D. thesis, California Institute of Technology, 2000.

[3] C. J. Hood et al., Science 287, 1447 (2000); Phys. Rev. A 63, 013401 (2001).

[4] P.W. H. Pinkse et al., Nature (London) 404, 365 (2000).

[5] G. R. Guthöhrlein et al., Nature (London) 414, 49 (2001).

[6] J. Eschner et al., Nature (London) 413, 495 (2001).

[7] T. Pellizari et al., Phys. Rev. Lett. 75, 3788 (1995).

[8] J. I. Cirac et al., Phys. Rev. Lett. 78, 3221 (1997).

[9] S. van Enk et al., Science 279, 205 (1998).

[10] C. Cabrillo et al., Phys. Rev. A 59, 1025 (1999).

[11] S. Bose et al., Phys. Rev. Lett. 83, 5158 (1999).

[12] A. S. Parkins and H. J. Kimble, J. Opt. B Quantum Semiclassical Opt. 1, 496 (1999).

[13] H. J. Metcalf and P. van der Straten, Laser Cooling and Trappingy (Springer-Verlag, Berlin, 1999).

[14] C. J. Hood and C. Wood, as described by H. J. Kimble et al., in Laser Spectroscopy XIV, edited by (World Scientific, Singapore, 1999), p. 80.

[15] H. Katori et al., J. Phys. Soc. Jpn. 68, 2479 (1999); T. Ido et al., Phys. Rev. A 61, 061403 (2000).

[16] S. J. van Enk et al., Phys. Rev. A 64, 013407 (2001).

[17] C. J. Hood et al., Phys. Rev. A 64, 033804 (2001).

[18] H. Mabuchi et al., Opt. Lett. 21, 1393 (1996).

[19] Because of small stress-induced birefringence in the cavity mirrors, we align the directions of linear polarization along an axis that coincides with one of the cavity eigenpolarizations [17], denoted by $\hat{l}_{ \pm}$. For initial polarization along $\hat{l}_{+}$, measurements of FORT [probe] polarization along $\hat{l}_{-}$for the cavity output power $P$ give $P_{-} / P_{+}<0.02[0.002]$ for the FORT [probe] beam.

[20] The (incoherent) sum of the four intensities is $I_{4-5} \sim$ $60 \mathrm{~mW} / \mathrm{cm}^{2}$ for the cooling and $I_{3-3} \sim 40 \mathrm{~mW} / \mathrm{cm}^{2}$ for the repumping light, with uncertainties of roughly 2 times.

[21] C. J. Hood et al., Phys. Rev. Lett. 80, 4157 (1998).

[22] Specific examples of single-atom detection events are omitted here. For $\Delta_{p} \simeq-g_{0}$, the increases in cavity transmission are quite similar to those in Refs. [3,21], while for $\Delta_{p}=0$ the decreases are similar to those in Refs. [2,18], albeit now in the presence of the FORT.

[23] $\bar{N}$ is estimated from the mean number of atom transit events (of duration $\simeq 150 \mu \mathrm{s}$ ) during the interval $\simeq$ $10 \mathrm{~ms}$ from the falling MOT atoms, in the absence of trapping.

[24] T. A. Savard et al., Phys. Rev. A 56, R1095 (1997); C.W. Gardiner et al., ibid. 61, 045801 (2000).

[25] The predicted $\tau_{p}^{\text {radial }}>10^{4} \mathrm{~s}$.

[26] K. L. Corwin et al., Phys. Rev. Lett. 83, 1311 (1999).

[27] R. A. Cline et al., Opt. Lett. 19, 207 (1994).

[28] A. Kuhn et al., Phys. Rev. Lett. 89, 067901 (2002).

[29] D. Boiron et al., Phys. Rev. A 53, R3734 (1996), and references therein. 